\title{
ESPONDILODISCITES SÉPTICAS
}

\section{Diagnóstico e tratamento}

\author{
Francisco Sérgio C. Barros Leal', Oswaldo Inácio de Tella Jr.', \\ Antônio de Pádua Furquim Bonatelli, Marco Antônio Herculano, \\ Paulo Henrique Aguiar ${ }^{4}$
}

\begin{abstract}
RESUMO - Estudamos retrospectivamente 24 pacientes com espondilodiscite séptica de diferentes etiologias (hematogênica, primária e infantil) e os diferentes aspectos envolvidos em seu diagnóstico e tratamento. Constatamos que a velocidade de hemossedimentação é um bom parâmetro laboratorial para acompanhar a evolução da doença, mas deve ser sempre interpretada conjuntamente com o quadro clínico e os achados de neuroimagem. Biópsias devem ser reservadas para os casos de diagnóstico duvidoso e o tratamento clínico realizado sempre que afastadas as seguintes condições: sepse, déficit neurológico, deformidade severa, abscesso epidural e corpo estranho (discite primária). A abordagem cirúrgica deve ser planejada levando em conta o estágio da doença, sendo preferencialmente por via posterior nas fases supurativas e anterior nas demais. Baseados em nossa experiência e em revisão da literatura, propomos um algoritmo para orientar o diagnóstico e o tratamento das espondilodiscites sépticas.
\end{abstract}

PALAVRAS-CHAVE: discite séptica, espondilodiscite séptica, osteomielite vertebral.

\section{Septic spondylodiscitis: diagnosis and treatment}

\begin{abstract}
We studied retrospectively 24 patients with septic discitis of different etiologies (hematogenic, primary and infantile) and the different aspects involved in its diagnosis and treatment. Erythrocyte sedimentation rate proved to be a valuable parameter and should always be interpreted carefully along with the clinical and neuroimaging findings. Biopsies should be reserved for doubtful cases with atypical course. Clinical treatment should be initiated after the following situations have been ruled out: sepsis, neurological deficit, severe deformity, epidural abscess and foreign body (primary disease). The surgical approach may be chosen based on the stage of disease, being preferably posterior in suppurative forms and anterior in the nonsuppurative stage. Based on our experience and on information gathered by literature review, we propose an algorithm to guide diagnosis and treatment in patients with septic discitis.
\end{abstract}

KEY WORDS: septic discitis, septic spondilodiscitis, vertebral osteomyelitis.

A espondilodiscite séptica ainda representa um desafio ao médico, quanto ao seu diagnóstico e tratamento. A literatura neurocirúrgica é muito escassa sobre o assunto e a maioria das publicações está distribuída entre especialidades como ortopedia, clínica geral, reumatologia, pediatria e radiologia. Embora sua fisiopatologia não seja completamente esclarecida, a maioria dos trabalhos é retrospectiva, formas diferentes da doença sendo agrupadas das mais diversas maneiras. Termos como espondilodis- cite, discite infantil e osteomielite vertebral são empregados como doenças diferentes, não se fazendo distinção entre adultos e crianças de diferentes idades e a inexistência de uma classificação universalmente aceita torna difícil a interpretação dos dados obtidos.

A modalidade de tratamento é igualmente controversa. A velocidade de hemossedimentação (VHS) é geralmente empregada para acompanhar a evolução da doença. Contudo, além de inespecífica, pode

Disciplina de Neurocirurgia da Escola Paulista de Medicina - Universidade Federal de São Paulo (UNIFESP) São Paulo SP, Brasil: ${ }^{1}$ Médico Pós-Graduando; ${ }^{2}$ Professor Adjunto Livre Docente; ${ }^{3}$ Professor Adjunto; ${ }^{4}$ Professor Colaborador da Disciplina de Neurocirurgia da Faculdade de Medicina da Universidade da São Paulo (FMUSP), São Paulo SP, Brasil.

Recebido 12 Dezembro 2002, recebido na forma final 7 Abril 2003. Aceito 24 Abril 2003.

Dr. Francisco Sérgio C. Barros Leal - Clínica Neurológica - Rua Conselheiro Moreira de Barros 159/17 - 12010-080 Taubaté SP - Brasil. FAX: 12 232.7874. E-mail: fcsergio@tuol.com.br 
levar muitos meses para normalizar, ou até mesmo ser normal no início da doença. Uma vez que a VHS encontra-se geralmente alterada durante algumas semanas após cirurgias assépticas da coluna vertebral, são necessários outros parâmetros para diagnosticar precocemente a infecção, pois as alterações radiológicas podem ser tardias e difíceis de interpretar no pós-operatório. A proteína C-reativa (PCR) é descrita como valiosa em diferenciar formas sépticas das assépticas; contudo, não sabemos ao certo até que ponto tal informação é confiável. Em vista das discrepâncias acima, realizamos um estudo retrospectivo de 24 pacientes com espondilodiscite séptica e analisamos os resultados após rever toda a literatura pertinente.

Nossos objetivos foram: 1) delinear uma abordagem sistemática e confiável para pacientes com espondilodiscite séptica; 2 ) avaliar as diferentes formas de tratamento; 3 ) verificar se a VHS é um bom parâmetro para suspender o tratamento antimicrobiano.

\section{MÉTODO}

Estudamos retrospectivamente 24 pacientes com espondilodiscite séptica infantil, primária e hematogênica, com idades entre um e 78 anos, analisando aspectos clínicos, laboratoriais, achados de neuroimagem, tratamento e complicações.

A distribuição dos pacientes quanto ao sexo, idade e forma da doença, são ilustrados na Tabela 1. O acompanhamento médio foi de 28,4 meses ( 0 - 91 meses), com nove pacientes $(37,5 \%)$ sendo entrevistados por ocasião dessa publicação (Figura 1).

Foram consideramos curados os pacientes cuja VHS normalizou ao final do tratamento ou quando esta caiu para um terço do valor inicial, desde que a duração do tratamento antimicrobiano tenha sido de no mínimo quatro meses (adultos) e com sinais radiológicos sugestivos de cicatrização do espaço discal. Consideramos melhorados os pacientes que evoluíram satisfatoriamente do ponto de vista clínico e laboratorial, mas que não foram seguidos por tempo suficiente para constatarmos a cura.
A classificação adotada foi a de Ring et al. ${ }^{1}$, onde são reconhecidas quatro formas da doença: neonatal, infantil, hematogênica e primária. A forma neonatal, por tratar-se de entidade distinta das demais, não está incluída no presente estudo.

\section{RESULTADOS}

A distribuição dos pacientes quanto ao sexo, idade e forma da doença é mostrada na Tabela 1. A duração média dos sintomas foi de três semanas nas formas infantis (2 - 4 semanas), com 9,3 semanas nas hematogênicas ( 2 - 28 semanas) e 8,4 semanas nas primárias ( 1 - 28 semanas).

Observamos que nas formas infantil e primária predominaram as lesões da coluna lombar, enquanto as osteomielites vertebrais acometeram principalmente a coluna lombar (60\%), seguida pela tóracolombar (20\%), cervical (13\%) e torácica (7\%). Entre as lesões lombares (18 pacientes), houve a predominância do espaço L5/S1 (44,4 \%), seguida por L4/ L5 (33,3 \%), L3/L4 (16,7 \%) e L3/L4/L5 (5,6 \%).

Os valores da VHS no inicio e no final do tratamento são resumidos na Tabela 2 .

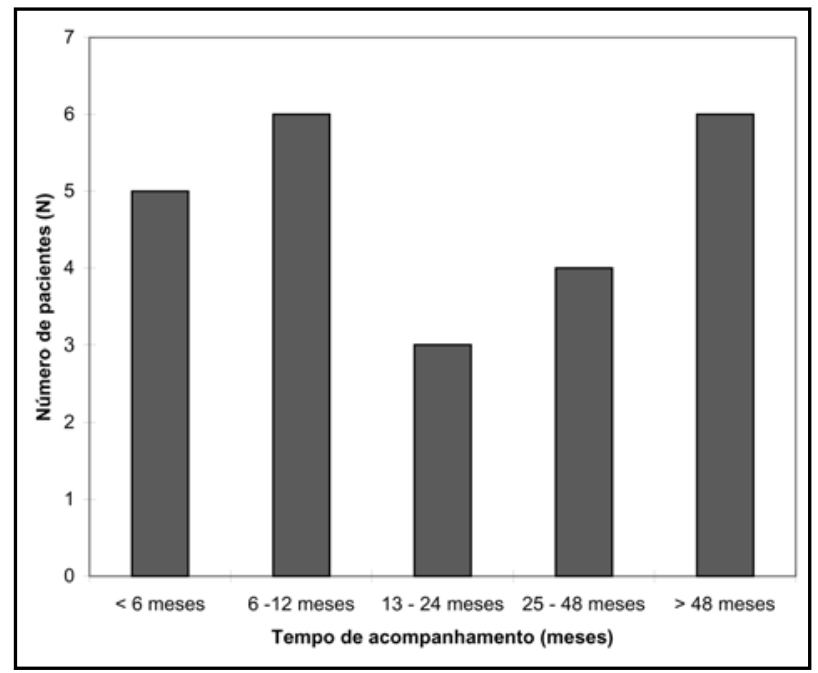

Fig 1. Distribuição dos pacientes quanto ao tempo de acompanhamento $(N=24)$.

Tabela 1. Distribuição dos pacientes de acordo com a forma da doença, sexo e idade.

\begin{tabular}{lccccccc}
\hline & & \multicolumn{3}{c}{ Sexo } & & Idade (anos) \\
\cline { 5 - 7 } $\begin{array}{l}\text { Forma da } \\
\text { doença }\end{array}$ & $\begin{array}{c}\text { Número de } \\
\text { pacientes }\end{array}$ & $\%$ & Masculino & $\%$ & Feminino & $\%$ & $\begin{array}{c}\text { Média } \\
\text { (mín-máx) }\end{array}$ \\
\hline $\begin{array}{lccccccc}\text { Infantil } \\
\text { Hematogênica }\end{array}$ & 4 & 16.7 & 2 & 50 & 2 & 50 & $3(1-7)^{*}$ \\
Primária & 15 & 62.5 & 11 & 73.3 & 4 & 26,7 & $54.8(35-67)$ \\
\hline
\end{tabular}

(*) Três crianças com idade igual ou inferior a dois anos (75\%). 
Tabela 2. Distribuição dos pacientes conforme a forma da doença, valores da VHS no início e no final do tratamento e tempo médio para a sua normalização.

\begin{tabular}{lcc}
\hline & $\begin{array}{c}\text { VHS inicial } \\
(\mathrm{mm} / \mathrm{h})\end{array}$ & $\begin{array}{c}\text { Tempo médio para } \\
\text { normalização da VHS } \\
(\text { semanas })\end{array}$ \\
Forma da doença $(\mathrm{n} / \mathrm{N})$ & Valor médio (min. -máx.) & \begin{tabular}{c} 
Valor médio (mín. -máx.) \\
\hline Infantil (4/4)
\end{tabular} \\
Hematogênica (10/15) & $73(12-109)$ & $11(10-12)$ \\
Primária $(5 / 5)$ & $56(10-121)$ & $16,8(4-28)$ \\
\hline
\end{tabular}

Máx, máximo; Mín, mínimo; n, número de pacientes cuja VHS foi determinada até o final do tratamento; N, número total de pacientes; VHS, velocidade de hemossedimentação. (*) Ao final do tratamento não houve normalização da VHS em dois pacientes com espondilodiscite primária e um com hematogênica e não foi determinada em duas crianças e quatro adultos com espondilodiscite hematogênica.

Tabela 3. Sensibilidade dos exames de neuroimagem inicialmente.

\begin{tabular}{|c|c|c|c|c|c|}
\hline & $\begin{array}{l}\text { Radiografia } \\
\text { simples } \\
(n=13)\end{array}$ & $\begin{array}{c}\text { Tomografia } \\
\text { computadorizada } \\
(n=12)\end{array}$ & $\begin{array}{c}\text { Ressonância } \\
\text { magnética } \\
(n=18)\end{array}$ & $\begin{array}{l}\text { Cintilografia } \\
\text { Tecnécio } \\
(n=7)\end{array}$ & $\begin{array}{c}\text { Gálio } \\
(n=2)\end{array}$ \\
\hline$N=24$ & $78,6 \%$ & $91,7 \%$ & $88,9 \%$ * & $83,3 \%$ & $50 \%$ \\
\hline
\end{tabular}

(*) Ressonância magnética foi inicialmente negativa em um paciente e apenas diagnosticou discite quando repetida uma semana após, elevando a sensibilidade do exame para $94,5 \%$. N, Número total de pacientes; $n$, Número de pacientes submetidos a cada exame.

A bactéria mais frequentemente isolada foi o Staphylococus aureus, exceto nas discites hematogênicas onde predominaram os Gram negativos. As sensibilidades das culturas obtidas foram as seguintes: sangue $=87,5 \%$; urina $=100 \%$; biópsia (osso ou disco) $=29 \%$; abscesso $=100 \%$; pus coletado na ferida cirúrgica $=100 \%$. Nos casos em que realizamos biópsia, a histologia evidenciou reação inflamatória crônica inespecífica em todos os casos. A sensibilidade dos exames de neuroimagem é mostrada na Tabela 3.

Dezenove pacientes foram curados $(79,2 \%)$, dois melhoraram (8,3 \%) e três morreram (12,5\%). Quatro pacientes desenvolveram déficit neurológico $(16,7 \%)$ : três tiveram déficit motor completo (1 tetraplegia e 2 paraplegias crurais) e um teve déficit incompleto (paraparesia crural com força muscular grau IV) (Tabela 4). O tratamento cirúrgico foi empregado em sete pacientes, todos adultos ( $29 \%$ ).

\section{DISCUSSÃO}

A espondilodiscite séptica é infecção bacteriana do espaço intervertebral e pode afetar pessoas de qualquer idade e de ambos os sexos. A infecção primária geralmente ocorre após discectomias ou procedimentos diagnósticos invasivos no disco. A forma hematogênica ou osteomielite vertebral é nor-
Tabela 4. Distribuição dos pacientes de acordo com a evolução e presença de déficit neurológico.

\begin{tabular}{lcc}
\hline Evolução final & $\begin{array}{c}\text { Número de } \\
\text { pacientes (\%) }\end{array}$ & $\begin{array}{c}\text { Déficit } \\
\text { neurológico (\%) }\end{array}$ \\
\hline Cura & $19(79,2 \%)$ & $2(8,3 \%)$ \\
Melhora & $2(8,3 \%)$ & - \\
Morte & $3(12,5 \%)$ & $2(8,3 \%)$ \\
Total & $24(100 \%)$ & $4(16,7 \%) *$ \\
\hline
\end{tabular}

(*) Todos com espondilodiscite hematogênica.

malmente causada por disseminação bacteriana por via sanguínea (embolização arterial). Esta, quando ocorre na infância, é denominada espondilodiscite infantil. Embora as três formas de espondilodiscite sejam classificadas como doenças diferentes, alguns autores não fazem qualquer distinção entre adultos e crianças de diferentes idades e aceitam todas como uma única entidade'.

A espondilodiscite infantil é de etiologia incerta, mas é geralmente aceita como benigna e autolimitada ${ }^{2,3}$; contudo, alguns casos podem se comportar como osteomielite vertebral, com quadro arrastado, destruição óssea e formação de abscessos 
(epidural, psoas, retrofaríngeo, ilíaco e paravertebral) ${ }^{1,3}$. Entre as possíveis etiologias estão as infecções bacterianas, virais, trauma e processos inespecíficos $^{1,4}$. Antecedentes de traumatismos podem ser obtidos em um terço dos casos, já tendo sido aventada a possibilidade de discite asséptica por liberação de fosfolipase $\mathrm{A}^{3}$.

Nas crianças, meninos e meninas são igualmente afetados, ocorrendo distribuição etária bimodal, com um primeiro pico entre seis meses e quatro anos de idade e um segundo, entre 10 e 14 anos $^{5}$. O segmento mais comumente afetado é o lombar, seguido pelo torácico e o cervical, podendo acometer dois ou mais níveis adjacentes ${ }^{6,7}$.

Os sintomas nas formas infantis geralmente iniciam agudamente e estão associados a doença sistêmica. A criança geralmente apresenta febre, irritabilidade, recusa-se a ficar de pé ou sentar e não consegue deambular. Em crianças abaixo de três anos é pouco provável encontrar queixas de lombalgia. Entre adultos é mais comum o início gradual dos sintomas (frequentemente acima de três meses) e predominam queixas de lombalgia e dor à palpação do local acometido, com limitação importante da movimentação devido ao espasmo muscular.

Em nosso estudo, os resultados foram semelhantes aos observados na literatura, não havendo prevalência de sexo, sendo $75 \%$ das crianças com idade inferior a dois anos e todos os casos acometendo a coluna lombar. A duração média dos sintomas foi de três semanas nas espondilodiscites infantis e 3,8 semanas nas primárias $(p>0,05)$, enquanto nas hematogênicas foi 9,3 semanas ( $p>0,05$ quando comparada com as formas anteriores). Três das quatro crianças desenvolveram sintomas sépticos e melhoraram rapidamente com o uso de antimicrobianos intravenosos. Achados semelhantes são descritos por outros autores ${ }^{3,8}$.

As osteomielites vertebrais ocorrem entre 1: 100000 e 1: 25000 da população adulta e representam 4 a $16 \%$ de todas as infecções ósseas de origem hematogênica ${ }^{9-11}$. Essas normalmente são de início insidioso e costumam ocorrer após bacteremias de repetição em pacientes debilitados e podem levar à morte se não tratadas adequadamente ${ }^{9,11}$. Tais sintomas constitucionais ocorreram em $60 \%$ de nossos pacientes.

Habitualmente existe predominância do sexo masculino nessa forma de infecção $(1,2: 1-4: 1)$, assim como distribuição etária bimodal, com um primeiro pico abaixo dos 20 anos e um segundo entre
50 e 70 anos $^{12,13}$. Entre 41 e $75 \%$ das infecções ocorrem em adultos acima de 50 anos ${ }^{11,14}$ e predominam na coluna lombar ${ }^{15,16}$. Os resultados que obtivemos relativos à distribuição por sexo, idade e localização estão resumidos na Tabela 1 e estão em conformidade com os achados dos referidos autores.

Vários são os fatores relacionados ao surgimento das espondilodiscites hematogênicas: diabetes mellitus, artrite reumatóide, uso de drogas endovenosas, uso de corticóides e alcoolismo ${ }^{8}$. Antecedentes de infecções são obtidos entre 20 e $82 \%$ dos $\operatorname{casos}^{11,14}$ e as fontes mais comuns de bactérias são as do trato gênito-urinário (13-61\%), partes moles (5-40\%), aparelho respiratório (5-40\%) e antecedentes de cirurgias (14$20 \%$ ), além de outros possíveis focos como endocardite, artrites e infecções de vias aéreas superiores ${ }^{8}$. Em 33 a $65 \%$ dos casos é possível identificar uma infecção concomitante fora da coluna vertebral ${ }^{11,17}$.

Entre os pacientes que estudamos, identificamos fatores de risco em 10 casos (67\%): diabetes mellitus (4), endocardite bacteriana (3), úlcera infectada em membro (2), infecção urinária (2), manipulação dentária (1) e alcoolismo (1), com alguns pacientes possuindo mais de um fator de risco.

As osteomielites são geralmente causadas por uma única bactéria, sendo o $S$. aureus o mais frequentemente isolado ${ }^{12,14,18}$. Entre as culturas obtidas também houve predominância de $S$. aureus (6), seguido de E. coli (3), S. bovis (1), P. mirabilis (1), P. aeruginosa (2) e $S$. epidermidis (1). Ambas as culturas com $P$. aeruginosa foram isoladas do mesmo paciente. A sensibilidade das culturas de urina, abscesso e secreção obtida da ferida cirúrgica foi $100 \%$, enquanto das hemoculturas foi $87,5 \%$ e apenas $29 \%$ em biópsias; contudo, a histologia sugeriu o diagnóstico em todos os casos (reação inflamatória crônica inespecífica).

Entre os cinco pacientes com espondilodiscite primária, todos foram operados uma única vez através de técnica microcirúrgica, sem artrodeses ou instrumentações. Todos tiveram infecções na coluna lombar $(\mathrm{L} 3 / \mathrm{L} 4=2, \mathrm{~L} 4 / \mathrm{L} 5=1, \mathrm{~L} 5-\mathrm{S} 1=2)$. Dois pacientes eram diabéticos (40\%) estando um deles em uso de corticóides para artrite reumatóide associada. Apenas dois pacientes (40\%) receberam antibioticoterapia peri-operatória.

Entre os achados radiológicos mais característicos estão a diminuição do espaço discal, que pode ser observada em radiografias simples entre duas e quatro semanas após o início dos sintomas e esclerose reativa de platôs vertebrais adjacentes (oito 
semanas), neoformação óssea (12 semanas) e fusão óssea (seis meses) ${ }^{9}$. A tomografia computadorizada (TC) mostra lesões líticas em espaço discal e abscessos paravertebrais, epidurais e no músculo psoas ${ }^{20}$. A ressonância magnética (RM) tipicamente revela destruição dos platôs discais, associada a hipossinal em T1 e hipersinal em T2 nos corpos vertebrais envolvidos, alem de diagnosticar eventuais lesões paravertebrais. As cintilografias se alteram mais precocemente que os exames anteriores e são particularmente úteis nos casos de curta duração, com sensibilidade de $90 \%$ e $92 \%$ e especificidade de $78 \%$ e $100 \%$ (tecnécio e gálio, respectivamente) ${ }^{21}$.

A sensibilidade dos nossos exames de neuroimagem foi semelhante à descrita por outros autores para as radiografias simples, RM e cintilografia com tecnécio, sendo inferior em relação à cintilografia com gálio e superior quando comparada à $\mathrm{TC}^{20,21}$. A pequena sensibilidade que encontramos nas cintilografias com gálio pode ser explicada pela pequena quantidade de exames realizados (apenas duas), sem falso-positivos (Tabela 3).

A VHS é o exame mais útil para diagnóstico e acompanhamento das discites, podendo estar normal em alguns casos. Seu valor se eleva antes de haver qualquer alteração radiológica e é o primeiro exame laboratorial a responder ao uso de antibióti$\cos ^{22}$. O método Westergren é o mais empregado, com valores normais inferiores a $15 \mathrm{~mm} / \mathrm{h}$.

Em nossa casuística a VHS estava elevada por ocasião do diagnóstico em $95,5 \%$ dos casos. O único paciente com VHS normal teve sua infecção diagnosticada somente através de cintilografia com gálio (espondilodiscite primária). Não foi possível determinar a VHS inicial em dois pacientes com espondilodiscite hematogênica, pois o primeiro já estava fazendo uso de antibióticos algumas semanas antes do diagnóstico e o outro faleceu de sepse dois dias após ser submetido a cirurgia de urgência para drenagem de empiema epidural torácico.

A VHS inicial, o tempo médio para a sua normalização e a duração da antibioticoterapia, foram significativamente menores nas formas infantis (Tabela 2) sugerindo melhor resposta ao tratamento e meIhor evolução $(p<0,05)$, sendo tal resultado também descrito por outros autores ${ }^{8,23}$. Ao compararmos as formas adultas, tais resultados não foram significativos $(p>0,05)$. A proteína C-reativa (PCR) foi determinada em apenas nove de nossos pacientes e estava elevada em oito (88,9\%).

Segundo Wenger et al. ${ }^{7}$ o tratamento antimicro- biano deve ser empregado em todos os casos de discite infantil. Contudo, outros autores recomendam a mesma apenas em casos com evolução não satisfatória ou sinais de infecção sistêmica ${ }^{24}$. Todas as crianças que estudamos apresentaram toxemia por ocasião do diagnóstico e receberam antimicrobianos por período médio de 6,75 semanas (2 - 12 sem.), evoluindo satisfatoriamente.

Nas espondilodiscites hematogênica e primária a duração média do tratamento antimicrobiano foi 17,2 semanas ( 1 - $28 \mathrm{sem}$.) e 17,6 semanas (10 - $28 \mathrm{sem}$.), respectivamente $(p>0,05)$. Tais resultados são semelhantes aos obtidos por Osenbach et al. ${ }^{14}$ Alguns autores acreditam que antibioticoterapia endovenosa inferior a quatro semanas nas formas adultas está relacionada a maior risco de recidiva da infecção ${ }^{14}$.

A VHS ao final do tratamento normalizou ou caiu a níveis próximos dos normais em todas as crianças, em $80 \%$ das discites primárias e $58 \%$ das hematogênicas. Entre os casos com elevação persistente da VHS, esta oscilou entre $22 \%$ e $83 \%$ dos valores iniciais. De acordo com Osenbach et al. ${ }^{14}$ a VHS nas discites hematogênicas deve cair para cerca de 25 $\mathrm{mm} / \mathrm{h}$ ao final do tratamento e nos casos em que não há normalização, ocorre redução de cerca de $50 \%$ do valor inicial em $94 \%$ dos casos, o que está de acordo com nossos achados.

Segundo Dauch ${ }^{19}$, parece haver aumento crescente na incidência das discites primárias na segunda metade do século passado, provavelmente devido a melhores meios diagnósticos $(0,2 \%$ da década de $50,0,8 \%$ na de 60 e $1,4 \%$ dos anos 70 ). Ao que parece, o traumatismo cirúrgico (principalmente quando utilizadas curetas), reoperações e restos de tecido necrótico no espaço discal agem como "locus minoris resistentiae" e favorecem ao alojamento e proliferação de bactérias ${ }^{19}$.

Entre os casos que operamos (cinco adultos), somente um foi reoperado, sendo isolado $S$. epidermidis do espaço discal. Todos foram submetidos a discectomias assépticas da coluna lombar. Dois desses pacientes eram diabéticos (40\%) e um outro estava em uso de corticosteróides para artrite reumatóide. Apenas dois pacientes receberam antibioticoterapia peri-operatória (40\%). Embora El-Gindi et al. ${ }^{23}$ sejam favoráveis à exploração cirúrgica em todos os casos de discite pós-operatória, Thibodeau ${ }^{25}$ considera duvidoso o tratamento cirúrgico precoce.

Entre os pacientes com espondilodiscite hematogênica, seis foram operados, sendo um desses devido ao diagnóstico inicial errôneo de hérnia de 


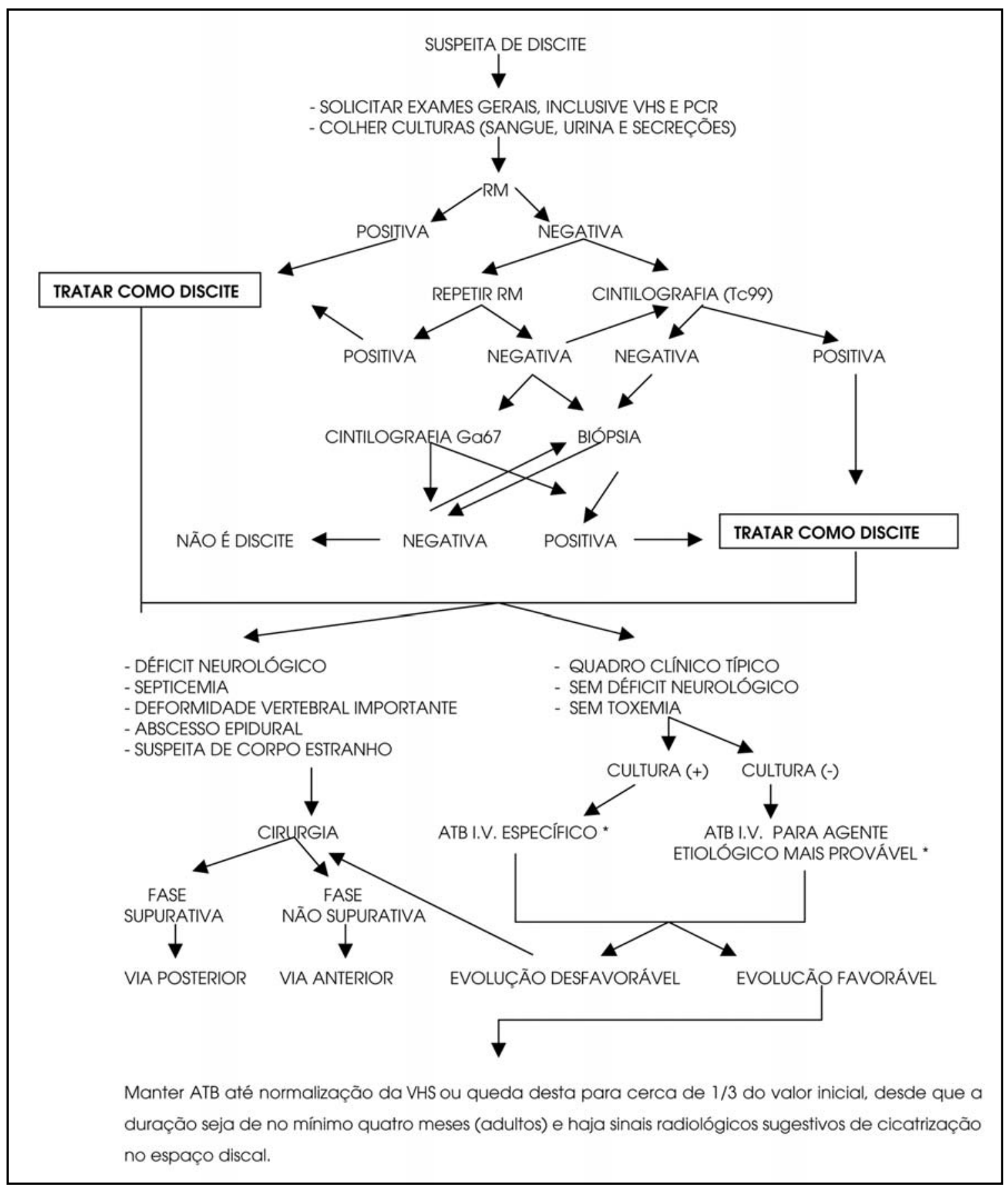

Fig 2. Algoritmo para diagnóstico e tratamento das espondilodiscites sépticas. (*) ATB I.V, antibioticoterapia intravenosa.

disco. Os demais foram operados pelas seguintes causas: duas laminectomias descompressivas (uma de urgência para drenagem de abscesso epidural e outra que resultou em hematoma epidural pós-operatório e paraparesia sequelar), uma artrodese com instrumentação por via posterior em T10/T11 para correção de deformidade, duas biópsias a céu aberto e uma descompressão transoral de C2 seguida de artrodese posterior com instrumentação occipitocervical.
Ocorreu déficit neurológico em quatro pacientes $(16,7 \%)$ (Tabela 4), todos com espondilodiscite hematogênica e lesões acima de T12, enquanto Silverthorn e Gillespie ${ }^{11}$ descreveram ocorrência de apenas 7\%. Nosso índice de déficit neurológico é baixo quando comparado aos resultados de Eismont ${ }^{18}$, que relata $51 \%$ de pacientes com algum tipo de déficit; contudo, estudou apenas lesões hematogênicas e incluiu casos de infecções fúngicas em seu estudo. 
Morreram três pacientes com espondilodiscite hematogênica; entretanto, apenas dois destes de causa relacionada à doença (o terceiro morreu de coleperitônio após alta hospitalar).

Tendo em vista as considerações acima, propomos o algoritmo para diagnosticar e tratar as espondilodiscites sépticas (Fig 2).

\section{CONCLUSÕES}

Baseados em nossa experiência e após revisão da literatura, concluímos que:

1. As discites infantis, primárias e hematogênicas representam provavelmente uma mesma doença, sendo a forma infantil a mais benigna, de tratamento mais simples e melhor prognóstico. A espondilodiscite hematogênica representa o outro extremo do espectro, com morbidade e mortalidade mais elevadas, diagnóstico tardio e frequentemente associada a co-morbidades como diabetes mellitus, alcoolismo e imunodepressão.

2. A VHS é o parâmetro laboratorial mais útil no diagnóstico das espondilodiscites sépticas e seus valores devem ser interpretados com cautela, conjuntamente com o quadro clínico e alterações neurorradiológicas. Uma VHS inicial normal não descarta a doença e antibióticos endovenosos devem ser administrados até a normalização ou redução da VHS para cerca de $1 / 3$ do valor inicial, desde que a duração do tratamento antimicrobiano seja de no mínimo quatro meses e haja sinais radiológicos de cicatrização do espaço discal.

3. Culturas devem ser obtidas de todos os possíveis focos de infecção sendo as biópsias reservadas para os casos duvidosos ou atípicos ou que não respondem adequadamente ao tratamento. Devem ser solicitadas culturas apropriadas para aeróbicos, anaeróbicos, fungos e micobactérias.

4. O tratamento conservador deve ser tentado inicialmente em todos os casos, exceto nas seguintes situações: sepse, déficit neurológico progressivo, deformidade espinhal importante, empiema epidural ou na possibilidade de corpo estranho pósoperatório. A abordagem cirúrgica deve levar em consideração o estágio da doença, sendo preferencialmente por via posterior nas fases supurativas e anterior nas demais.

\section{REFERÊNCIAS}

1. Ring D, Johnston CE, Wenger DR. Pyogenic infectious spondydilitis in children: the convergence of discitis and vertebral osteomyelitis. J Pediatr Orthop 1995;15:652-660.

2. Atar D, Lehman WB, Grant AD. Discitis in children. Orthop Rev 1992;21:931-933.

3. Cushing AH. Diskitis in children. Clin Infect Dis 1993;17:1-6.

4. Alexander $\mathrm{O}$. The etiology of 'juvenile spondyloarthritis (discitis). Clin Radiol 1970;21:178-186.

5. Mandeil GA, Harcke Hl. Scintigraphy of spinal disorders in adolescents. Skeletal Radiol 1993;22:393-401.

6. Ryöppy 5, Jääskelläinen J, Rapola J, Alberty A. Nonspecific diskitis in children. A nonmicrobial disease ? Clin Orthop 1993;297:95-99.

7. Wenger DR, Bobechko WP, Gilday DL. The spectrum of intervertebral discspace infection in children. J Bone Joint Surg 1978;60:100-108.

8. Przybylski O, Nelson PB. Vertebral osteomyelitis and disc space infections. In: Tindall CI, Cooper PR, Barrow DL. (EDS) The practice of neurosurgery. Baltimore: Williams \& Wilkins, 1996:3467-3479.

9. Digby JM, Kersley JB. Pyogenic non-tuberculous spinal infection: an analysis of thirty cases. J Bone Joint Surg 1979;61:47-55.

10. Kulowski J. Pyogenic osteomyelitis of the spine: an analysis and discussion of 102 cases. J Bone Joint Surg 1936;18:343-364.

11. Silverthorn KG, Gillespie WJ. Pyogenic spinal osteomyelitis: a review of 61 cases. N Z Med J 1986;99:62-65.

12. Bonfiglio M, Lange IA, Kim YM. Pyogenic vertebral osteomyelitis. Clin Orthop 1973;96:234-247.

13. Meyers SP, Wiener SN. Diagnosis of hemogenous pyogenic vertebral osteomyelitis by magnetic resonance imaging. Arch Intern Med 1991; 151:683-687.

14. Osenbach RK, Kitchon PW, Menezes AH. Diagnosis and management of pyogenic vertebral osteomyelitis in adults. Surg Neurol 1990;33:266-275.

15. Garcia A Jr, Grantham AS. Hematogeneous pyogenic vertebral osteomyelitis. J Bone Joint Surg 1960;42:429-436.

16. Ross PM, Fleming JL. Vertebral osteomyelitis: spectrum and natural history. A retrospective analysis of 37 cases. Clin Orthop 1976;118:190-198.

17. Hitchon PW, Osenbach RK, Yuh WTC, Menezes AH. Spinal infections. Clin Neurosurg 1992;38:373-387.

18. Eismont FJ, Bohlman HH, Soni PL, Goldberg VM, Freehafer AA. Pyogenic and fungal vertebral osteomyelitis with paralysis. J Bone Joint Surg 1983;65:19-29.

19. Dauch WA. Infection of the intervertebral space following conventional and microsurgical operation of the herniated lumbar intervertebral disk: a controlled clinical trial. Acta Neurochir(Wien) 1986;82:43-49.

20. Kopecky KK, Gilmor RL, Scott JA, Edward AM. Pitfalls of computed tomography in diagnosis of discitis. Neuroradiology 1985;27:57-66.

21. Modic MT, Feiglin DH, Piraino DW, et al. Vertebral osteomyelitis: assessment using MR. Radiology 1985;157:157-166.

22. Barros Leal, FSC. Aspectos envolvidos no diagnóstico e tratamento das discites sépticas. Tese. Escola Paulista de Medicina, Universidade Federal de São Paulo. São Paulo, 1999.

23. El-Gindi S, Aref S, Salama M, Andrews J. Infection of intervertebral disc after operation. J Bone Joint Surg 1976;58:114-116.

24. Smith RF, Taylor TKF. Inflammatory lesions of intervertebral disks in children. J Bone Joint Surg 1967;49:1508-1520.

25. Thibodeau AA. Closed space infection folowing removal of lumbar intervertebral disc. J Bone Joint Surg 1968;50:400-410. 